\title{
Metaphysical Indeterminacy and Worldly Incompleteness
}

\begin{abstract}
Alessandro Torza
Abstract

An influential theory has it that metaphysical indeterminacy occurs just when reality can be made completely precise in multiple ways. That characterization is formulated by employing the modal apparatus of ersatz possible worlds. As quantum physics taught us, reality cannot be made completely precise. I meet the challenge by providing an alternative theory which preserves the use of ersatz worlds but rejects the precisificational view of metaphysical indeterminacy. The upshot of the proposed theory is that it is metaphysically indeterminate whether $p$ just in case it is neither true nor false that $p$, and no terms in ' $p$ ' are semantically defective. In other words, metaphysical indeterminacy arises when the world cannot be adequately described by a complete set of sentences defined in a semantically nondefective language. Moreover, the present theory provides a reductive analysis of metaphysical indeterminacy, unlike its influential predecessor. Finally, I argue that any adequate logic of a language with an indeterminate subject matter is neither compositional nor bivalent.
\end{abstract}

\section{Precisificational possibilities}

The idea that indeterminacy may be not only semantic (originating in language ${ }^{1}$ ), but also metaphysical (originating in the nonrepresentational

\footnotetext{
${ }^{1}$ The dominant theory of semantic indeterminacy is the supervaluationism of Fine [16].
} 
world), has been a fringe view until not long ago, mostly due to a combination of two factors: an influential argument of Evans [15] against vague objects, ${ }^{2}$ as well as a lack of theories that could capture metaphysical indeterminacy in a clear and rigorous fashion. The tide turned with a number of recent papers which have brought the notion of metaphysical indeterminacy out of disrepute. I will focus on the most discussed proposal, developed by Elizabeth Barnes and Robert Williams (henceforth, BW). ${ }^{3}$

The BW account models metaphysical indeterminacy in terms of precisifications: roughly, the world is indeterminate just in case there are multiple ways it can be made precise. The idea is spelled out by way of the standard distinction between a world, a concrete object made up of things and properties, and an ersatz world, an abstract representation of a world. For our present purpose, we can assume linguistic ersatzism, the view that what does the representing are maximally consistent sets of sentences defined in a semantically nondefective world-making language - although nothing essential hinges on the linguistic construal of ersatz worlds. That the world-making language is semantically nondefective means that none of its terms are either vacuous or semantically vague. (The assumption of semantic nondefectiveness is crucial in distinguishing metaphysical indeterminacy from other kinds of indeterminacy, as will be discussed in section 2.) BW assume that the ersatz worlds are maximally consistent with respect to a background classical logic. ${ }^{4}$

\footnotetext{
${ }^{2}$ The target of Evans' argument is indeterminate identity. While his result does not per se rule out the possibility of metaphysical indeterminacy sans indeterminate identity, as a matter of fact it had the effect of putting metaphysical indeterminacy in a bad light overall.

${ }^{3}$ The most developed version of the account is Barnes and Williams [7]; see also Barnes [5], Williams [25] [26]. The version of BW's account which I employ in the present discussion is based on Williams [26]. Alternative accounts of metaphysical indeterminacy include Akiba [1] [2], Wilson [27]; see Bokulich [10] for a discussion of quantum metaphysical indeterminacy.

${ }^{4}$ Barnes and Williams [7, p. 114]. The classical discussion of ersatzism, linguistic and otherwise, is Lewis [17, pp. 136-91].
} 
Following BW, an ersatz world $E$ is said to be a precisificational possibility for a world $w$ if $E$ does not determinately misrepresent $w$-formally, $\forall p(p \in E \rightarrow$ ' $p$ ' is not determinately false at $w)$. A world $w$ is said to be metaphysically indeterminate if it has multiple precisificational possibilities. ${ }^{5}$ Notice that BW's proposal does not amount to an analysis of metaphysical indeterminacy, since the definiens employs the notion of determinacy. The resulting theory is therefore unlike standard supervaluationism, which analyzes semantic indeterminacy in terms of quantification over (admissible) precisifications.

An ersatz world is said to be a precisificational actuality if it is a precisificational possibility for the actual world. So, our world is metaphysically indeterminate just in case there are multiple precisificational actualities. For instance, consider the classical problem of material constitution involving a statue (Goliath) and a lump of clay (Lumpl). Some regard them as one object having this or that modal property relative to a mode of presentation, or to a standard of similarity by which counterparts are picked out. An alternative view has it that the statue and the lump of clay are distinct but spatiotemporally coincident objects. Both views are currently regarded as live theoretical possibilities, and both are presumably consistent with the empirical data. I submit that the controversy could be defused by diagnosing it as an instance of metaphysical indeterminacy, to wit as a case in which things are such that it is indeterminate whether the statue and the lump of clay are identical. The BW theory allows us to make that intuition precise: metaphysical indeterminacy about the identity of Lumpl and Goliath amounts to the existence of (at least) two precisificational actualities, one containing the sentence 'Lumpl is Goliath', one containing the sentence 'Lumpl is not Goliath'.

\footnotetext{
${ }^{5}$ Akiba [3] argued that BW's notion of metaphysical indeterminacy is a consequence of uncontroversial facts about indeterminacy, and therefore that there is nothing specifically metaphysical about it. I will come back to this issue in section 2 .
} 
Skow [23] has argued that there are instances of deep metaphysical indeterminacy which cannot be adequately modeled in terms of the BW account, due to the fact that reality cannot be made completely precise (cf. Darby [13]). Examples of deep metaphysical indeterminacy can be cooked up by appealing to quantum mechanics. According to Heisenberg's uncertainty principle, there are pairs of quantum mechanical properties (observables) which are incompatible, in the sense that they cannot both have determinate values for a given particle at the same time. For instance, an electron cannot have both determinate position and determinate momentum at any given time - the more precise the position, the less precise the momentum, and vice versa. Now, suppose that electron $e$ has position values $x_{i \in I}$ and momentum values $p_{j \in J}$, where $I, J$ are uncountable sets of indices. Let $\phi\left(e, x_{i}\right)$ stand for ' $e$ has position $x_{i}$ ', and $\chi\left(e, p_{j}\right)$ stand for ' $e$ has momentum $p_{j}$ '. If, at some time $t$, it is true that $\chi\left(e, p_{j}\right)$, for some $j \in J$, it follows by the uncertainty principle that $\phi\left(e, x_{i}\right)$ is untrue, for all $i \in I$. On the hypothesis that quantum indeterminacy is metaphysical in character, ${ }^{6} \mathrm{BW}$ tell us that there are uncountably many precisificational actualities $E_{i \in I}$ such that $\left\{\chi\left(e, p_{j}\right), \phi\left(e, x_{i}\right)\right\} \subseteq E_{i}$, for all $i \in I$. But we know from the uncertainty principle that position and momentum cannot both be sharp; therefore there are no such possible ersatz worlds as $E_{i}$, against the BW account. It must be concluded that the BW account is unable to model quantum metaphysical indeterminacy.

Before I turn to discussing the objection from deep metaphysical indeterminacy, one caveat is in order. When I speak of quantum indeterminacy, I do not mean that it may be indeterminate which state of a quantum system is actualized. That is, of course, determinate: the actual state is the one picked out by the system's wave function. What I mean, instead, is that a particle may fail to have a determinate (or definite, or sharp) value of some quantum property. In other words, quantum indeterminacy is property in-

\footnotetext{
${ }^{6}$ The thesis that quantum mechanics provides examples of metaphysical indeterminacy has been articulated in Bokulich [10], Lowe [18] [19], Williams [25], Wilson [27].
} 
determinacy, rather than state indeterminacy. The key issue of this paper is to determine whether property indeterminacy at the quantum level can be subsumed by a general theory of metaphysical indeterminacy in the vicinity of BW.

Two reactions to Skow's argument come immediately to mind. First of all, one might think that the quantum formalism is incomplete, and that the uncertainty principle expresses a merely epistemic constraint. If so, fundamental physics need not be a source of metaphysical indeterminacy, thus leaving the BW account unscathed. In such a scenario, it would be up to the physicists to formulate a hidden variable theory, i.e., a framework which matches the predictive power of standard quantum theory, and explains away the uncertainty in epistemic terms. ${ }^{7}$

Now, the orthodox (Copenhagen) interpretation of quantum mechanics regards the formalism as complete, thus ruling out the possibility of hidden variables. But what if the orthodox interpretation turns out to be incorrect? Skow $[23$, p. 856$]$ points out that the orthodox interpretation is at least possibly true, which suffices to show the inadequacy of the BW account, since the latter aims to model metaphysical indeterminacy at every possible world. One might rejoin that, when the orthodox interpretation (or any interpretation of quantum mechanics, for that matter) is said to be possible, the relevant notion of possibility is epistemic. For when we say of some theory that it might be true, what is meant is that, for all we know, the actual world is the way that theory prescribes. But a thesis which is epistemically possible need not be true at some world, as we know from

\footnotetext{
${ }^{7}$ There exist results - most notably Bell's theorem and the Kochen-Specker theoremthat impose strict constraints on any empirically adequate hidden variable theory. It is a consequence of the Kochen-Specker theorem that there is no hidden variable formulation of quantum mechanics, as long as the value of an observable is independent of how that value is measured. Bell's theorem states that any hidden variable theory must be nonlocal, allowing some sort of action at a distance.
} 
the existence of metaphysically impossible propositions which used to be epistemic possibilities - e.g., that Hesperus is not Phosphorus. Be that as it may, I will set this objection aside and concede to Skow that the orthodox interpretation is a bona fide metaphysical possibility.

The second reaction is that the objection from deep metaphysical indeterminacy hinges on the requirement that every $E_{i}$ is a possible ersatz world. If we generalize the BW account by allowing impossible ersatz worlds (contradictory sets of sentences closed under some paraconsistent logic), we could capture the indeterminacy of $e$ 's position, as desired. I think there are two problems with that proposal. One is methodological. Sure, it has been argued that impossibilia play a significant role in metaphysical theorizing, especially in modeling fine-grained properties and propositions, making sense of counterpossible reasoning, as well as providing a modal characterization of essence (Berto [8], Broogard and Salerno [11], Correia [12], Nolan [20], Vander Laan [24], Yagisawa [29]). Nevertheless, ontological parsimony and theoretical conservativity advise caution: the addition of impossibilia is only justified if strictly required, ceteris paribus. As I will argue in the next section, we can model quantum metaphysical indeterminacy without bothering with impossible ersatz worlds.

One may rejoin that every $E_{i}$ is physically impossible but metaphysically possible, and therefore that we can accept it in our ontology without the slightest form of revisionism. The move, however, is fallacious. Since precisificational actualities are complete descriptions, they must contain a sentence stating Heisenberg's uncertaintity principle, which is logically inconsistent with each $\left\{\chi\left(e, p_{j}\right), \phi\left(e, x_{i}\right)\right\}$. Thus, no $E_{i}$ is metaphysically possible.

A further reason for being skeptical about the impossible-world strategy is the following. Consider the aforementioned interpretation of the puzzle of material constitution in terms of metaphysically indeterminate identity. If the precisificational actualities are all of the ersatz worlds, possible or 
impossible, which do not determinately misrepresent how things are, one of such ersatz worlds will have to be an impossible one containing both 'Lumpl is Goliath' and 'Lumpl is not Goliath'. Consequently, there is a way to make actuality precise in which a contradiction is true, which means that the actual world is not determinately possible. But such a conclusion is overly revisionary, insofar as every theory of modality (perhaps with the sole exception of dialetheism) regards the actual world as determinately possible. As long as we want a theory of metaphysical indeterminacy which is compatible with the standard view of actuality as an instance of possibility, the impossible-world approach ought to be deemed inadequate.

\section{Incomplete ersatz worlds}

In the previous section I introduced the BW account of metaphysical indeterminacy, and explained why that account is unable to capture quantum metaphysical indeterminacy. The gist of the objection is that not all metaphysical indeterminacy can be understood in terms of ways the world can be made completely precise. Skow [23, p. 858] concludes that "How to model deep metaphysical indeterminacy remains an open question." In the present section I will sketch an alternative framework which does away with the precisificational approach, while preserving the machinery of ersatz possible worlds. The upshot will be a view according to which metaphysical indeterminacy arises just when some sentence, defined in a semantically nondefective language, is neither true nor false.

One moral that can be drawn from the discussion of the previous section is that we should reject the received view that logical space is defined by the maximally consistent sets of sentences. In order to see why, let us go back to our electron $e$. We saw that a precisificational possibility $E_{k}$ for that world cannot contain any $\phi\left(e, x_{i}\right)$, if it contains some $\chi\left(e, p_{j}\right)$. Since BW's theory assumes that ersatz worlds are maximal, each $E_{k}$ will then have to contain 
every negation $\neg \phi\left(e, x_{i}\right)$, which leads to trouble. Recall that our goal is to model the fact that, when momentum is sharp, every atomic position statement is untrue, that is:

(i) $e$ does not have a determinate position.

However, if every $\neg \phi\left(e, x_{i}\right)$ is in each $E_{k}$, then all atomic position statements are false, and so:

(ii) $e$ does not have a position.

For the statement that $e$ has a position is regimented in terms of a sentence of the form $\exists z \phi(e, z)$. Assuming that $x_{i \in I}$ are all the position values, the statement is logically equivalent with $\bigvee_{i \in I} \phi\left(e, x_{i}\right)$, which is the infinitary disjunction of the sentences expressing the possible determinate positions of $e$. If each disjunct $\phi\left(e, x_{i}\right)$ is false, $\bigvee_{i \in I} \phi\left(e, x_{i}\right)$ is false too (by De Morgan's Laws), and so is $\exists z \phi(e, z){ }^{8}$

Incidentally, notice that if we do embrace (ii), a response to Skow's objection is available on behalf of BW. For the argument from deep metaphysical indeterminacy first assumes that quantum indeterminacy is a particular case of metaphysical indeterminacy; then shows that quantum indeterminacy cannot be modeled via BW's theory; and finally concludes that not all metaphysical indeterminacy can be modeled via BW's theory. Of course, if (ii) was the case, quantum indeterminacy would not be an instance of metaphysical indeterminacy (since any statement about position, or what have you, would be determinately true or determinately false), thus making Skow's argument unsound.

As it turns out, there are empirical reasons for believing that superposition of position states entails indeterminate position (as per (i)), rather than lack of position (as per (ii)). The crucial fact is that particles can be at a determinate distance from each other, even when their positions are indeterminate. That would be impossible, if superposition of position states

\footnotetext{
${ }^{8}$ A classical reference on infinitary logic is Dickmann [14].
} 
amounted to a lack of position - provided that being at a determinate distance from something presupposes having a position. Therefore, it is correct to say that a particle can have indeterminate position, but not that it can have no position.

Let's unpack the argument. As a preliminary remark, it is noteworthy that a particle in a superposition of position states can have a determinate distance from another particle:

Suppose an electron and a proton become entangled in the position degree of freedom. It may be possible to ascribe to the two-particle system a definite difference of positions $\left(x_{A}-x_{B}\right)$, without being able to ascribe a definite position to either particle individually. For example, the particles might determinately be ten meters apart, even though neither particle by itself has a definite position. (Bokulich [10, p. 469-70])

But only something with a position can be at a determinate distance from something else. As a consequence, particles with indeterminate position can have a position, contra (ii).

The above line of thought can be distilled into the following argument:

1. Physical distance is a relation between positions in physical space (or spacetime).

2. Distance between physical objects is physical distance between their positions.

3. Therefore, physical objects which are at some distance from each other are objects which have a position in space (or spacetime).

4. Particles with indeterminate position can be at some distance from each other.

5. Therefore, particles with indeterminate position can have a position in space (or spacetime). 
I regard premises (1) and (2) as definitionally true; premise (4) is an empirical fact, as remarked; and the inference is valid. Therefore, thesis (ii) is false.

Let's take stock. We have just seen that there are empirically informed reasons for rejecting (ii). But (ii) is a consequence of the assumption that ersatz worlds are maximal. Therefore, we should embrace the revisionary view that regards ersatz worlds as consistent, but possibly incomplete sets of sentences in a world-making language. ${ }^{9}$

My goal, for the remainder of this section, is to show that metaphysical indeterminacy can be successfully captured by means of incomplete ersatz worlds. The solution, I submit, is to abandon the notion of a precisificational possibility altogether and define the theory as follows. An ersatz world $E$ is said to be an adequate possibility for a world $w$ if $E$ represents $w$-formally, $\forall p(p \in E \leftrightarrow$ ' $p$ ' is true at $w)$. A world $w$ is said to be metaphysically indeterminate if it has an incomplete adequate possibility. An ersatz world is said to be an adequate actuality if it is an adequate possibility for the actual world. So, our world is metaphysically indeterminate just in case there is an incomplete adequate actuality.

The resulting picture is rather straightforward: a world is metaphysically determinate when it is adequately represented by a maximally consistent ersatz world; it is indeterminate otherwise, which is to say, when it is adequately represented by some gappy ersatz world.

The intuition underlying the present proposal is quite natural. BW and I agree on one thing: for reality to be determinate is to be described by a unique and complete ersatz world. According to BW, what fails in cases of indeterminacy is the uniqueness condition. The BW characterization, however, is undermined by cases of deep metaphysical indeterminacy. On the present proposal, it is the completeness condition that fails in cases of in-

\footnotetext{
${ }^{9}$ Cf. Putnam [22, p. 185]: "A system has no complete description in quantum mechanics; such a thing is a logical impossibility.".
} 
determinacy. In other words, reality is metaphysically indeterminate when some sentence formulated in a semantically nondefective language is neither true nor false. As I will soon argue, the present characterization avoids the issue of deep metaphysical indeterminacy.

A few remarks are in order. First of all, since ersatz worlds can be incomplete, the definition of an adequate possibility has to involve a biconditionalunlike BW's definition of a precisificational possibility. For if we had defined an adequate possibility by means of the clause ' $\forall p\left(p \in E \rightarrow{ }^{6} p\right.$ ' is true at $\left.w\right)$ ', it would follow that the empty set is an adequate possibility for any world, and therefore that metaphysical indeterminacy is trivially necessary.

Secondly, every world has exactly one adequate possibility. For suppose that some world $w$ is represented by distinct $E, E^{\prime}$. By extensionality, there would have to be some sentence $p$ which is in $E$ but not $E^{\prime}$ (or vice versa), and therefore $p$ would be both true and untrue at $w$, a contradiction.

Thirdly, and most importantly, the present proposal yields a reductive analysis of the notion of metaphysical indeterminacy — unlike BW's account. Indeed, whereas the notion of determinacy is employed in the definition of a precisificational possibility (see previous section), it is not employed in the definition of an adequate possibility. In particular, the present proposal reduces metaphysical indeterminacy to representational incompleteness in a semantically nondefective language.

Back to the objection from deep metaphysical indeterminacy, it is easy to see that the problem is quickly taken care of. To say that electron $e$ has determinate momentum and indeterminate position is tantamount to saying that the one adequate actuality contains $\chi\left(e, p_{j}\right)$, for some $j \in J$, but neither $\phi\left(e, x_{i}\right)$ nor its negation, for some $i \in I$. This fact is not only consistent with Heisenberg's uncertainty principle - it also meets the desideratum that our metaphysics be inspired by the current best science. For if quantum theory (according to the orthodox interpretation) tells us that determinacy 
of all observables is impossible, it is methodologically wise to rule out the existence of (possible) ersatz worlds where quantum theory is true and some complementary observables, such as position and momentum, are both defined for the same particle at the same time.

The present proposal seems to face an objection from expressive incompleteness. Let $w$ be a quantum-mechanical world containing only one particle: an electron $e$, which has neither determinate position nor determinate momentum. (The quantum formalism doesn't allow complementary observables to be both sharp, but it allows them to be both unsharp.) Since the adequate possibility $E$ for $w$ won't contain $\chi\left(e, p_{j}\right), \phi\left(e, x_{i}\right)$ or their negations, $E$ must be the empty ersatz world. ${ }^{10}$ But then there are multiple possible states of $e$, in fact infinitely many, which are represented by the empty ersatz world - some of them are states of $e$ in which the position is sharper and the momentum is less sharp, whereas others are states of $e$ in which the momentum is sharper and the position is less sharp. As a consequence, there is a one-many correspondence between ersatz worlds and possibilities. Logical space is incomplete, and badly so.

The objection fails because it unduly assumes that position and momentum are the only observables expressible in the world-making language. Sure, if the world-making language were to be that impoverished, it should be no wonder that logical space turns out to be incomplete. But it is a fact about quantum mechanics that every state of a system is sharp with respect to some observable. (Formally: every vector in a Hilbert space is an eigenvector of some Hermitian operator. ${ }^{11}$ ) We may assume that to every

\footnotetext{
${ }^{10} \mathrm{I}$ am here ignoring the fact that $E$ must include general information about $w$, such as the quantum-mechanical laws, as well as particular information about the intrinsic properties of $e$, and some necessary truths.

11 "Any Hermitian operator on a given space will invariably be associated with some measurable property of the physical system connected with that space... Any vector whatever in a given space will invariably be an eigenvector of some complete Hermitian operator on that space. That... will entail that any quantum state whatever of a given
} 
quantum observable there corresponds a predicate in the world-making language. As a consequence, the state of our electron $e$ in $w$ will be expressed by some sentence ' $s$ ' $=$ ' $e$ has property $P$ with value $x$ '; and so the adequate possibility $E$ for $w$ will have ' $s$ ' as a member. The moral is that, provided that the world-making language is sufficiently rich, to every state of every (quantum) world there will correspond a sentence which appears as a member of the relevant adequate possibility, in such a way that to different states there correspond different adequate possibilities. The present proposal has not been shown to suffer from expressive incompleteness. ${ }^{12}$

A second objection is that the present view overgenerates instances of metaphysical indeterminacy. Akiba [3] has argued that the BW account collapses metaphysical indeterminacy with indeterminacy simpliciter. For, goes the objection, on the BW account metaphysical indeterminacy arises iff the world has multiple precisificational possibilities; which is the case iff physical system will invariably be associated with some definite value of some measurable property of that system." Albert [4, p. 41].

${ }^{12}$ One may rejoin that my reply conflates Hermitian operators with properties. For, goes the objection, the fact that every quantum property is captured by some Hermitian operator by no means entails that every Hermitian operator captures some quantum property. Consequently, I have failed to show that every state of a system is sharp with respect to some property. (I owe this observation to an anonymous referee.) The objection can be dealt with by appealing to the standard distinction between an abundant and a sparse conception of properties. When the objector raises doubts about the claim that every Hermitian operator captures some quantum property, what she has in mind is arguably the sparse conception of a physical property, or observable. The idea is that, even though one can define infinitely many Hermitian operators in a Hilbert space, it is highly doubtful that to each there corresponds some genuine physical property. But of course I do not need to endorse such a preposterous view. The notion of property, or observable, that I presuppose in my reply to the objection from expressive incompleteness is the abundant one, according to which a property is the semantic value of a condition definable in the relevant language. In the present case the language is the language of quantum mechanics, and the semantic values of the conditions definable in that language are Hermitian operators. Therefore, there is no risk of running out of properties in the relevant sense. 
some sentence $p$ is such that neither $p$ is false nor $\neg p$ is false; which is the case iff some sentence $p$ is neither true nor false; which is the case iff it is indeterminate whether $p$, for some $p$. It follows that the BW theory is unable to characterize metaphysical indeterminacy as a peculiar phenomenon differing from semantic indeterminacy (or any kind of indeterminacy originating in the representational world).

Akiba's objection in fact carries over to all accounts of metaphysical indeterminacy based on ersatz worlds. As for my own account, the argument would go thus: on the present view metaphysical indeterminacy arises iff the adequate ersatz world is incomplete; which is the case iff there is some $p$ such that neither $p$ nor $\neg p$ belongs to the adequate ersatz world; which is the case iff some $p$ is neither true nor false; which is the case iff it is indeterminate whether $p$, for some $p$.

However, recall a key assumption built into the way the BW account was introduced in section 1: the world-making language is semantically nondefective. Even though Barnes and Williams do not specify that condition in so many words, it is quite clear that they take their ersatz worlds (or whatever representational devices play the relevant role) to be precise, and that is the way the BW is typically understood in the literature. ${ }^{13}$ Precisely because of that feature, on the BW account the world has multiple precisificational possibilities iff some sentence $p$ is such that neither $p$ is false nor $\neg p$ is false and the language of $p$ is semantically nondefective; which is the case iff it is indeterminate whether $p$, for some $p$, and the language of $p$ is semantically nondefective. As a consequence, Akiba's purported rebuttal of the BW account misfires.

Since I am also assuming that the world-making language is semantically nondefective, Akiba's argument cannot be successfully deployed against my own view, and for the very same reason. Indeed, on the present proposal

\footnotetext{
${ }^{13}$ Cf. Skow [23, p. 858], who considers and rejects a modification of the BW account in which the ersatz worlds are defined in "a language which suffers from semantic indeterminacy".
} 
the adequate ersatz world is incomplete iff there is some $p$ such that neither $p$ nor $\neg p$ belongs to the actual ersatz world and the language of $p$ is semantically nondefective; which is the case iff it is indeterminate whether $p$, for some $p$, and the language of $p$ is semantically nondefective. It can be concluded that the present view does not collapse metaphysical indeterminacy with indeterminacy simpliciter.

Can we conclude that the present view does not overgenerate instances of metaphysical indeterminacy? Not quite. There are two special cases that need to be taken care of. First of all, one might point to some $p$ which is semantically indeterminate, even though the language of $p$ is semantically nondefective. A paradigmatic case is reference failure, as in 'the present king of France is a king'. Here, one might argue, we have a definite description built out of semantically nondefective terms: the descriptor 'the', and the complex predicate 'present king of France'. Nevertheless, the resulting description 'the present king of France' is empty, and so the whole sentence is truth-valueless. Therefore, my theory falsely entails that it is metaphysically indeterminate whether the present king of France is a king. (To avoid noise, I assume that all nonlogical constants in the sentence are semantically precise.) But of course, if the sentence is indeterminate, the indeterminacy does not originate in the nonrepresentational world, but rather in the semantics of 'the present king of France'.

I reply by denying that 'the present king of France is a king' is truthvalueless. There are known ways of articulating this view, which is essentially Russellian, so I will not rehearse them here. Nevertheless, I concede that the objection can be blocked only by taking a stance on the debate about the logical form of definite descriptions.

There is a second class of cases in which my theory may overgenerate. Consider the sentence 'Hesperus is a planet'. One might argue that the sentence is truth-valueless at any world $w$ where Hesperus does not exist. 
But the language in which the sentence is formulated is semantically nondefective. Therefore, my theory falsely entails that, at $w$, it is metaphysically indeterminate whether Hesperus is a planet. (Again, I assume that all nonlogical constants in the sentence are semantically precise.) But of course there is nothing about the nonrepresentational world $w$ which makes the sentence indeterminate. The reason of the indeterminacy is purely semantic, namely the fact that 'Hesperus' fails to refer at $w$.

I reply by denying that 'Hesperus is a planet' is truth-valueless at any world where Hesperus does not exist. There are routine ways of articulating the semantics so as to meet that constraint, namely by adopting a positive or negative semantics for free logic, so I will not rehearse them here. ${ }^{14} \mathrm{Nev}-$ ertheless, I concede that, in order to block the objection, I need to take a stance on the debate about the semantics of possibly nonreferring individual constants.

\section{Compositionality}

Having settled the problem of characterizing metaphysical indeterminacy, it remains to establish the logic of a language with an indeterminate subject matter, i.e., one able to express instances of metaphysical indeterminacy. Even though the task of providing an answer to that question lies beyond the scope of the present work, I am going to make a few remarks which will hopefully pave the way for identifying the correct logic. In particular, I am going to argue that the logic of metaphysical indeterminacy is noncompositional. I will restrict the scope of the discussion to basic propositional languages - although a fully satisfactory account should cover at least firstorder languages with operators for modality and determinacy (a task carried out in Barnes and Williams [7]).

I assume a modal account of logical validity for a basic propositional language: $p$ is said to be valid if it is true at all worlds. As a consequence, $p$ is

\footnotetext{
${ }^{14}$ On the topic of free logics and their semantics, see Nolt [21].
} 
valid iff it is a member of every ersatz world. First of all notice that, insofar as there are cases of metaphysical indeterminacy, actual or possible, and metaphysical indeterminacy is tantamount to truth-valuelesness of sentences of a certain kind, then the logic of a language with an indeterminate subject matter is not bivalent.

Moreover, in the previous section empirically informed reasons were provided in support of the view that the disjunction $\bigvee_{i \in I} \phi\left(e, x_{i}\right)$ of the sentences expressing all possible position states of a particle $e$ can be true, even when no disjunct is true. (The reason, once again, is that the disjunction is equivalent with the sentence ' $e$ has a position' (i.e., $\exists z \phi(e, z)$ ), which can be true even when none of its instances is.) That fact entails the following logical thesis: the disjunction of the propositions expressing all possible values of a given quantum observable for a particle at a time can be true, even when no disjunct is true. ${ }^{15}$

Now, let $e$ be in a superposition of position states. It follows that no $\phi\left(e, x_{i}\right)$ is true. We also know that, when $e$ is in a superposition of position states, it is possible that ' $e$ has a position' is true and so, by De Morgan's Laws, that some $\phi\left(e, x_{i}\right)$ is not false. Some $\phi\left(e, x_{i}\right)$ must therefore be indeterminate. Let's suppose that every $\phi\left(e, x_{i}\right)$ is indeterminate - to be sure, a physically possible scenario, namely one in which $e$ is not in an eigenstate of position, and the probability of $e$ to have any determinate position upon measurement is nonzero. Whatever logic turns out to be correct for a language with an indeterminate subject matter, it is to be expected that disjunction be idempotent, i.e., any sentence $p$ is equivalent with a (possibly infinitary) disjunction $p \vee p \vee \ldots$ It follows that the sentence obtained from $\bigvee_{i \in I} \phi\left(e, x_{i}\right)$ by replacing each disjunct $\phi\left(e, x_{i}\right)$ with the materially equivalent $\phi\left(e, x_{1}\right)$ is going to be indeterminate. On the other hand, $\bigvee_{i \in I} \phi\left(e, x_{i}\right)$

\footnotetext{
${ }^{15}$ Incidentally, that thesis is validated by the quantum logic of Birkhoff and von Neumann [9], where the behavior of sentential connectives is read off of the structure of Hilbert spaces. It is worth remarking, however, that my argument from the previous section concerning the truth value of $\bigvee_{i \in I} \phi\left(e, x_{i}\right)$ is independent of the acceptance of quantum logic.
} 
is by hypothesis true. It must be concluded that a logic of metaphysical indeterminacy which accounts for quantum phenomena is going to be noncompositional, since it does not permit substitution of materially equivalent sentences salva veritate. ${ }^{16}$

Albert [4, p. 38] has defended the view that when a particle is in a superposition of states relative to an observable, the predicate expressing that observable simply does not apply:

The right way to think about superpositions of, say, being black and being white is to think of them as situations wherein color predicates cannot be applied, situations wherein color talk is unintelligible. Talking and inquiring about the color of an electron in such circumstances is (on this view) like talking or inquiring about, say, whether or not the number 5 is still a bachelor. [...] And that's the way things are, on this view, for all sorts of superposition: superpositions are situations wherein the superposed predicates just don’t apply.

Is Albert's observation correct? Yes and no. Sure, every $\phi\left(e, x_{i}\right)$ is truthvalueless whenever a particle is in a superposition of all positions states; and the ersatz world which adequately represents that superposition will not have any $\phi\left(e, x_{i}\right)$ or its negation as members. Nevertheless, in virtue of the noncompositionality of the logic of indeterminacy, complex propositions built out of those atomic sentences - such as $\bigvee_{i \in I} \phi\left(e, x_{i}\right)$ - can still be true, and therefore be members of the relevant ersatz world.

\footnotetext{
${ }^{16}$ In BW's theory a sentence is valid just in case it is a member of all precisificational possibilities for all worlds. Since BW's ersatz worlds are classical maximally consistent sets of sentences, every classical tautology is valid. On the other hand, indeterminate sentences are neither true nor false. Thus, BW's logic is neither bivalent nor compositional. An important caveat: not all versions of BW's account have those features. In particular, the logic of indeterminacy developed in Barnes and Williams [7] is both bivalent and compositional.
} 
The present, noncompositional account of quantum metaphysical indeterminacy is to be distinguished from the account of Wilson [27] [28] and Bokulich [10]. According to Wilson, metaphysical indeterminacy obtains just when there is some object possessing a determinable property $P$ but no unique determinate of $P$. So, there are two kinds of metaphysical indeterminacy: the gappy cases, when something has a determinable property $P$ but no determinate of $P$; and the glutty cases, when something has a determinable property $P$ and multiple determinates of $P$. Wilson-Bokulich regard quantum indeterminacy as an instance of gappy metaphysical indeterminacy, since a quantum particle can have the determinable property of position (or momentum, etc.) but no determinate position value (or momentum value, etc.).

How does the Wilson-Bokulich construal of metaphysical quantum indeterminacy stack up against mine? On either proposal, it can be true that $e$ has a position, even when $e$ is in a superposition of position states. Now, recall that the statement that $e$ has a position is of the form $\exists z \phi(e, z)$, which is logically equivalent to $\bigvee_{i \in I} \phi\left(e, x_{i}\right)$, when $x_{i \in I}$ are all the position values. Wilson-Bokulich claim that, in cases of superposition, $\exists z \phi(e, z)$ is true whereas each $\phi\left(e, x_{i}\right)$ is false, insofar as $e$ determinately fails to have any particular position (i.e., any determinate of the determinable position). But since $\exists z \phi(e, z)$ is true, it cannot be the case that every $\phi\left(e, x_{i}\right)$ is false (by De Morgan's Laws). So, the Wilson-Bokulich view appears to be inconsistent, unless they can either (i) deny De Morgan's Laws, or (ii) deny the equivalence of $\exists z \phi(e, z)$ and $\bigvee_{i \in I} \phi\left(e, x_{i}\right)$, or (iii) deny that $e$ 's having a position is regimented by a sentence of the form $\exists z \phi(e, z)$.

Since Wilson claims that her theory is compatible with classical logic, (i) must be ruled out. The same can be said of (ii), insofar as denying the equivalence of $\exists z \phi(e, z)$ and $\bigvee_{i \in I} \phi\left(e, x_{i}\right)$ would be logically revisionary, albeit at the predicate, rather than propositional level. ${ }^{17}$ Regardless, I doubt

\footnotetext{
${ }^{17}$ Of course, $\exists z \phi(e, z)$ and $\bigvee_{i \in I} \phi\left(e, x_{i}\right)$ are not logically equivalent if the class of position values is contingent. But that issue is orthogonal to the problem being discussed. Thus,
} 
there is any well-motivated reason for taking on board either (i) or (ii). We are left with option (iii), which is to deny that ' $e$ has a position' is correctly regimented by a sentence of the form $\exists z \phi(e, z)$. The problem with this third route is that, according to the quantum mechanical formalism, ' $e$ has a position' displays precisely that quantifier structure. For in quantum mechanics position is captured by a position operator, which induces a partial function $\phi$ from particles to position values. (The function is partial because, in virtue of the eigenstate-eigenvalue link, only particles whose state is an eigenstate of the position operator are assigned a position value.) Therefore, for $e$ to have a position is for it to have assigned some position value $z$ under the function $\phi$, i.e. to be such that $\exists z \phi(e, z)$.

To conclude, Wilson-Bokulich have not provided a viable account of quantum metaphysical indeterminacy. For, on pain of inconsistency, they would have to embrace either an overly revisionary logic (options (i) and (ii)) or an understanding of physical determinables which flouts the quantum formalism (option (iii)). ${ }^{18}$

\section{Conclusions}

There was a time when the intelligibility of de re modality was cast into doubt, mainly due to the absence of a precise framework that would capture the peculiarity of its logic and semantics. With the advent of Kripke-style to avoid pointless complications, I am setting the issue aside.

${ }^{18} \mathrm{I}$ also have more general misgivings about Wilson's theory of metaphysical indeterminacy. First of all, it is unable to model metaphysically indeterminate existence (cf. Barnes and Cameron [6]), because it would require existence and nonexistence to be determinates of some determinable - an implausible view. Second, I think that none of the examples of glutty metaphysical indeterminacy offered in Wilson [27] are adequately motivated (cf. Bokulich [10]). Finally, Wilson regards open future claims as expressing instances of metaphysical indeterminacy. Although I agree with her on that, I doubt that indeterminacy about the open future can be given a determinable-based account (cf. Barnes and Cameron [6]). A comprehensive assessment of Wilson's theory of metaphysical indeterminacy goes beyond the scope of this paper. 
model theory, some common misconceptions were cleared up and modal predication became mainstream. Barnes and Williams, among others, have tried to do the same with the notion of metaphysical indeterminacy: make it respectable in the philosophical circles by spelling out its logical and semantic structure. The resulting theory, however, is unable to model deep metaphysical indeterminacy, due to the fact that quantum observables cannot be all assigned precise values at the same time. I argued that the problem of deep metaphysical indeterminacy can be defused by giving up the idea that ersatz possible worlds must be complete. A further advantage of the present proposal over BW is that it provides a reductive analysis of metaphysical indeterminacy. Finally, I have argued that the logic of indeterminacy is neither compositional nor bivalent. Which (nonclassical) logic is correct for modeling languages with an indeterminate subject matter remains an open question.

Acknowledgements. I would like to express my gratitude to Axel Barceló, Aldo Filomeno, Eduardo García-Ramírez, John Horden, Ricardo Mena, Elias Okon and Jessica Wilson for helpful and constructive feedback on previous versions of this paper. I also would like to thank the audience of the workshops Logical Space. Logical and metaphysical issues and Philosophical Aspects of Modality, which took place at the Instituto de Investigaciones Filosóficas, UNAM, in September 2016. This work was supported by the PAPIIT grant IA400316.

\section{References}

[1] Akiba, K. (2000). Vagueness as a modality. Philosophical Quarterly 50: 359-70.

[2] Akiba, K. (2004). Vagueness in the world. Noûs 38: 407-29.

[3] Akiba, K. (2015). How Barnes and Williams have failed to present an intelligible ontic theory of vagueness. Analysis, 75(4): 565-73. 
[4] Albert, D. Z. (2009). Quantum mechanics and experience. Harvard University Press.

[5] Barnes, E. (2010). Ontic Vagueness: A Guide for the Perplexed. Noûs, 44(4): 601-27.

[6] Barnes, E. and Cameron, R. (2009). The Open Future: Bivalence, Determinism and Ontology. Philosophical Studies, 146: 291-309.

[7] Barnes, E. and Williams, J. R. G. (2011). A Theory of Metaphysical Indeterminacy. In Zimmerman, D. and Bennett, K. (eds) Oxford Studies in Metaphysics, vol. 6: 103-48. Oxford: Oxford University Press.

[8] Berto, F. (2010). Impossible Worlds and Propositions: Against the Parity Thesis. The Philosophical Quarterly, 40: 471-86.

[9] Birkhoff, G., and von Neumann, J. (1936). The Logic of Quantum Mechanics. Annals of Mathematics, 37: 823-43.

[10] Bokulich, A. (2014). Metaphysical Indeterminacy, Properties, and Quantum Theory. Res Philosophica, 91(3), 449-75.

[11] Brogaard, B. and Salerno, J. (2013). Remarks on Counterpossibles. Synthese, 190: 639-60.

[12] Correia, F. (2007). (Finean) Essence and (Priorean) Modality. Dialectica, 61(1): 63-84.

[13] Darby, G. (2010). Quantum mechanics and metaphysical indeterminacy. Australasian Journal of Philosophy, 88(2): 227-45.

[14] Dickmann, M. A. (1975). Large infinitary languages: model theory (Vol. 83). Amsterdam: North-Holland.

[15] Evans, G. (1978). Can there be Vague Objects? Analysis, 38: 208.

[16] Fine, K. (1975). Vagueness, truth and logic. Synthese, 54: 235-59. 
[17] Lewis, D. (1986). On the plurality of worlds. Oxford: Blackwell.

[18] Lowe, E. J. (1994). Vague Identity and Quantum Indeterminacy. Analysis, 54 (2): 110-14.

[19] Lowe, E. J. (1999). Vague Identity and Quantum Indeterminacy: Further Reflections. Analysis, 59(264): 328-30.

[20] Nolan, D. (1997). Impossible Worlds: A Modest Approach. Notre Dame Journal of Formal Logic, 38: 535-72.

[21] Nolt, J. (2006). Free logics. In Jacquette, D. (ed.) Philosophy of Logic: Handbook of the Philosophy of Science. Amsterdam: North Holland.

[22] Putnam, H. (1979). The Logic of Quantum Mechanics. In: Mathematics, Matter and Method. Philosophical Papers Volume 1. Cambridge UP.

[23] Skow, B. (2010). Deep metaphysical indeterminacy. The Philosophical Quarterly, 60(241): 851-58.

[24] Vander Laan, D. (1997). The Ontology of Impossible Worlds. Notre Dame Journal of Formal Logic, 38: 597-620.

[25] Williams, J. R. G. (2008). Ontic vagueness and metaphysical indeterminacy. Philosophy Compass, 3(4): 763-88.

[26] Williams, J. R. G. (2008). Multiple actualities and ontically vague identity. The Philosophical Quarterly. 58(230): 134-54.

[27] Wilson, J. (2013). A Determinable-Based Account of Metaphysical Indeterminacy. Inquiry 56 (4): 359-85.

[28] Wilson, J. (forthcoming). Are There Indeterminate States of Affairs? Yes. In Barnes, E. (ed.) Current Controversies in Metaphysics. Taylor and Francis. 
[29] Yagisawa, T. (1988). Beyond Possible Worlds. Philosophical Studies, 53: $175-204$. 